\title{
Comparative Analysis of KP-HSA Complex by Spectroscopic Methods
}

\author{
M. MąciażeK-JurczyK*, J. Równicka-Zubik, R. Dyja And A. SuŁkowska \\ Department of Physical Pharmacy, Medical University of Silesia, Jagiellońska 4, 41-200 Sosnowiec, Poland
}

(Received August 9, 2012; in final form February 2, 2013)

The main objective of the presented study was to characterize the high (HAS) and low affinity (LAS) binding sites of ketoprofen (KP) in human serum albumin (HSA) structure with the use of spectrofluorescence and proton nuclear magnetic resonance spectroscopy. In vitro fluorescence analysis was used to estimate the effect of KP on the HSA fluorescence. The association constants $K_{\mathrm{a}}\left[\mathrm{M}^{-1}\right]$ of KP-HSA complex in the HAS were determined with the use of Scatchard, Klotz, and Hill analysis. The quenching $K_{\mathrm{Q}}\left[\mathrm{M}^{-1}\right]$ constants were determined on the basis of the Stern-Volmer equation. Binding of ketoprofen to plasma protein was also studied with the use of 8-anilinonapthalene-1-sulfonic acid (ANS) and 5-dimethylaminonaphthalene-1-sulfonic acid (DNSA) as the fluorescence probes in IIIA and IIA subdomains of HSA, respectively. To estimate the cooperativeness in proteins Hill's coefficient $n_{\mathrm{H}}$ was used. The analysis of proton nuclear magnetic resonance spectra of KP in the presence of HSA allows us to observe the interactions between aromatic rings of the drug and the rings of amino acids located in the hydrophobic subdomains of the protein on the basis of the changes of chemical shifts $\Delta \sigma$ [ppm] of drug protons resonances. Moreover the $K_{\mathrm{a}}$ constants $\left[\mathrm{M}^{-1}\right]$ of KP-HSA complex in the LAS were determined.

DOI: $10.12693 /$ APhysPolA.123.673

PACS: 33.50.-j, 33.50.Dq, 82.56.-b, 87.14.-g, 87.14.E-

\section{Introduction}

Rheumatoid arthritis (RA) is an autoimmune disease that causes chronic inflammation of the joints [1-3]. In the treatment of RA nonsteroidal anti-inflammatory drugs (NSAIDs) are used. NSAIDs are structurally diverse chemical class of drugs. They are characterized by identical pharmacological properties, mechanism of action and adverse side effects. The knowledge of the location of the binding sites of the ligands gives vital information concerning drug design. Ketoprofen (2-(3-benzoylphenyl)propanoic acid, KP) is the nonsteroidal anti-inflammatory drug used to treat RA. KP is transported in the body via the circulatory system bound with protein plasma in about $99 \%$ [4].

Human serum albumin (HSA), the most abundant plasma protein, binds a wide variety of hydrophobic ligands including e.g. fatty acids, bilirubin, thyroxin. HSA consists of a single polypeptide with 585 amino acids residues containing 17 pairs of disulfide bridges and one free cysteine. HSA is often used as a carrier molecule due to its binding capacity, and thus mainly functions as the regulator of the colloidal osmotic pressure of the blood $[5,6]$. Serum albumin consists of three homologous domains (I-III), each composed of two subdomains (A and B) $[7,8]$. Albumin has two specific drug binding sites with high affinity in the IIA and IIIA subdomain. The pocket of subdomain IIA seems to correspond to the so-called site I, which is thought to be a binding site for salicylates, sulfonamides and a number of other drugs [9]. The inside wall of the pocket is formed by hydrophobic side chains, whereas the entrance of the pocket is sur-

*corresponding author; e-mail: mmaciazek@sum.edu.pl rounded by positively charged residues, i.e., Arg 257 , Arg 222, Lys 199, His 242, Arg 218 and Lys 195. To site II aromatic carboxylic acids largely ionized at physiological $\mathrm{pH}$ are bound. Site II, the putative binding site for tryptophane, thyroxine, octanoate and other drugs, would correspond to the pocket of subdomain IIIA, which is almost the same size as site I. The pocket is lined by hydrophobic side chains and the double disulfide bridges of helix IIIA-h3. The side chain of Arg 410 is located at the mouth of the pocket while the hydroxyl of Tyr 411 faces toward the inside of the pocket [9].

Fluorescence technique allowed for observation of the high affinity interaction, characterized by slow exchange $[10,11]$ between drug and serum albumin (SA) in the primary binding site. Proton nuclear magnetic resonance $\left({ }^{1} \mathrm{HNMR}\right)$ technique is suitable for studies of the weak low affinity interaction. ${ }^{1} \mathrm{HNMR}$ parameters of the drug protons, such as chemical shifts are the weighted average of the free and bound states.

The binding of KP to HSA have been already studied using photoaffinity labeling technique [12] and site-directed mutagenesis [13]. These studies present molecular docking of KP to HSA. The presented study describes biding mode of KP to HSA. The main objective of the presented study was to characterize the high (HAS) and low affinity binding sites (LAS) of KP in HSA structure with the use of spectrofluorescence and ${ }^{1} \mathrm{HNMR}$ spectroscopy.

\section{Materials and methods}

\subsection{Reagents}

Racemic KP, 8-anilino-1-naphtalenesulfonic acid (ANS), deuterium oxide $\left(\mathrm{D}_{2} \mathrm{O}\right)$ were obtained from Sigma-Aldrich Chemical Co. Human albumin fraction V 
(HSA) was purchased from ICN Biomedicals INC., Aurora, OH, USA. 5-dimethylaminonaphthalene-1-sulfonic acid (DNSA) was purchased from Fluka.

\subsection{Fluorescent measurements}

All solutions were prepared by titration method at room temperature using $0.05 \mathrm{~mol} / \mathrm{L}$ sodium phosphate buffer ( $\mathrm{pH} 7.47 \pm 0.1$ ). Ketoprofen was initially dissolved in $0.001 \mathrm{~mol} / \mathrm{L} \mathrm{NaOH}$. The measurements were performed at $310 \mathrm{~K}$. Emission fluorescence spectra were recorded using Hitachi FL-2500 spectrophotometer with $1 \mathrm{~cm} \times 1 \mathrm{~cm} \times 4 \mathrm{~cm}$ quartz cells and slits of $5 \mathrm{~nm} / 5 \mathrm{~nm}$. The range of recorded spectra for $\lambda_{\mathrm{ex}}=295 \mathrm{~nm}$ was 295 $400 \mathrm{~nm}$. For the studies on interaction between KP and ANS in subdomain IIIA the $295 \mathrm{~nm}, 360 \mathrm{~nm}$ wavelengths were used to excite the ANS probe. To excite the DNSA, probe of the binding in subdomain IIA, the $350 \mathrm{~nm}$ wavelength was used. The range of recorded spectra for HSAANS(1:1)-KP and HSA-DNSA(1:5)-KP complexes was 400-540 $\mathrm{nm}$ and $400-600 \mathrm{~nm}$, respectively. The concentration range of ketoprofen used in the studies was $5 \times 10^{-6} \mathrm{~mol} / \mathrm{L}$ to $4 \times 10^{-4} \mathrm{~mol} / \mathrm{L}$ and the concentration of HSA was constant and equals to $8 \times 10^{-6} \mathrm{~mol} / \mathrm{L}$. Concentrations of ANS and DNSA were constant and equal to $8 \times 10^{-6} \mathrm{~mol} / \mathrm{L}$ and $4 \times 10^{-5} \mathrm{~mol} / \mathrm{L}$, respectively. Absorbance of KP, ANS and DNSA at the used concentration was $\leq 0.05$ and the fluorescence spectra have not been corrected for the inner filter effect [14].

\subsection{Determination of binding parameters}

If all fluorophores are accessible to a quencher, the fluorescence process can be described by the Stern-Volmer linear regression equation [15]:

$$
\frac{F_{0}}{F}=1+K_{\mathrm{Q}}[\mathrm{L}],
$$

where $F, F_{0}$ are the steady state fluorescence intensities of protein at the band maximum in the absence and presence of quencher, respectively; $K_{\mathrm{Q}}$ is the dynamic quenching constant, $[\mathrm{L}]$ is the total quencher concentration.

When the Stern-Volmer equation has the exponential dependence (upward or downward curvature in the Stern-Volmer plot), quenching fluorescence of serum albumin in the presence of the ligand can be characterized by a modified Stern-Volmer nonlinear regression equation, from which dynamic $K_{\mathrm{Q}}\left[\mathrm{M}^{-1}\right]$ and static quenching constant $V\left[\mathrm{M}^{-1}\right]$ can be determined [16]:

$$
\frac{F_{0}}{F}=\left(1+K_{\mathrm{Q}}[\mathrm{L}]\right) \mathrm{e}^{V[\mathrm{~L}]},
$$

where $F, F_{0}$ are the steady state fluorescence intensities of protein in the absence and presence of quencher, respectively; $K_{\mathrm{Q}}$ is the dynamic quenching constant; [L] is the total quencher concentration, $V$ is the static quenching constant.

The Stern-Volmer equation modified by Lehrer [17] was used to determine the quenching constant $K_{\mathrm{Q}}\left[\mathrm{M}^{-1}\right]$ and fractional accessible protein fluorescence $f_{\mathrm{a}}$ :

$$
\frac{F_{0}}{F_{0}-F}=\frac{1}{[\mathrm{~L}]} \frac{1}{f_{\mathrm{a}}} \frac{1}{K_{\mathrm{Q}}}+\frac{1}{f_{\mathrm{a}}},
$$

where $F_{0}, F$ are the relative fluorescence intensity of protein in the absence and presence of quencher $(L)$, respectively; [L] is the quencher concentration; $f_{\mathrm{a}}$ is the fraction of the initial fluorescence accessible to quenching; $K_{\mathrm{Q}}$ is the quenching constant.

Association constants $K_{\mathrm{a}}\left[\mathrm{M}^{-1}\right]$ of ketoprofen to protein were evaluated using Scatchard- (Eq. (2.4)), Klotz(Eq. (2.5)) and Hill- (Eq. (2.6)) linear regression analysis.

Association $K_{\mathrm{a}}\left[\mathrm{M}^{-1}\right]$ for $n=1$ was determined with the use of the Scatchard method modified by Hiratsuka [18]:

$$
\frac{r}{\left[\mathrm{~L}_{\mathrm{f}}\right]}=n K_{\mathrm{a}}-K_{\mathrm{a}} r
$$

where $r$ is the number of ligands bound to one protein molecule; $\left[\mathrm{L}_{\mathrm{f}}\right]$ is the free (unbound) ligand concentration; $n$ is the number of binding sites; $K_{\mathrm{a}}$ is the association constant.

From the Klotz equation association constant was also determined [19]:

$$
\frac{1}{r}=\frac{1}{n}+\frac{1}{n K_{\mathrm{a}}} \frac{1}{\left[\mathrm{~L}_{\mathrm{f}}\right]}
$$

where $r$ is the number of moles of ligand bound per mole of protein; $n$ is the number of binding sites; $K_{\mathrm{a}}$ is the association constant; $\left[\mathrm{L}_{\mathrm{f}}\right]$ is the concentration of the free (unbound) ligand.

Hill's coefficient $n_{\mathrm{H}}$ and association constant $K_{\mathrm{a}}\left[\mathrm{M}^{-1}\right]$ were determined graphically on the basis of the following equation [20]:

$$
\log \left(\frac{Q}{1-Q}\right)=n_{\mathrm{H}} \log K_{\mathrm{a}}+n_{\mathrm{H}} \log \left[\mathrm{L}_{\mathrm{f}}\right],
$$

where $Q=F / F_{\max }$ is the fractional binding saturation, fraction of sites occupied with the ligand; $n_{\mathrm{H}}$ is the Hill coefficient; $K_{\mathrm{a}}$ is the association constant.

This form of Hill's equation is used to estimate the cooperativity in multisubunits' allosteric proteins.

\subsection{Proton magnetic resonance ${ }^{1} H N M R$ measurements}

All stock solutions of KP and HSA were prepared in $0.05 \mathrm{~mol} / \mathrm{L}$ sodium phosphate buffer $(7.4 \pm 0.1)$ in $\mathrm{D}_{2} \mathrm{O}$. Ketoprofen was initially dissolved in $0.001 \mathrm{~mol} / \mathrm{L} \mathrm{NaOD.}$ Final concentration of $\mathrm{KP}$ and $\mathrm{HSA}$ was $1 \times 10^{-3}$ to $8 \times 10^{-2}$ and $2.5 \times 10^{-4} \mathrm{~mol} / \mathrm{L}$, respectively. All measurements were carried out at $310 \mathrm{~K} .{ }^{1} \mathrm{HNMR}$ spectra were recorded on a Bruker Avance 400 spectrometer, probe was tuned at $400 \mathrm{MHz}$ and $5 \mathrm{~mm}$ tubes were used. For water suppression the presaturation method was used. Chemical shifts of KP proton resonance signals were measured in relation to the DSS signal at $0.015 \mathrm{ppm}$. Maximum error of chemical shift and relaxation time were $0.0001 \mathrm{ppm}(0.001 \mathrm{~Hz})$ and $0.001 \mathrm{~s}$, respectively. For the analysis the $\mathrm{H} 16$ and $\mathrm{H} 8$ proton resonances were chosen. The chemical shifts $\sigma$ [ppm] of proton resonances of KP in the KP-HSA binary system were compared with those of KP in the buffer. Then $\Delta \sigma \mathrm{ppm}$ values were calculated. The proton resonance assignment was performed 
on the basis of the simulated spectra of ketoprofen obtained with the use of $\mathrm{ACD} /{ }^{1} \mathrm{HNMR}$ Predictor (v7.07) and with MestRe-C 2.3a software.

\subsection{Determination of association constants}

Association constant $K_{\mathrm{a}}\left[\mathrm{M}^{-1}\right]$ was determined on the basis of the following equation [21]:

$$
\frac{1}{\sigma-\sigma_{\mathrm{f}}}=\frac{1}{\left(\sigma_{\mathrm{b}}-\sigma_{\mathrm{f}}\right)[\mathrm{P}]_{0}}\left([\mathrm{~L}]_{0}+K_{\mathrm{d}}\right),
$$

where $[\mathrm{L}]_{0}$ is the total ligand concentration; $[\mathrm{P}]_{0}$ is the total protein concentration; $K_{\mathrm{d}}$ is the dissociation constant.

\section{Results and discussion}

\subsection{Fluorescence studies}

3.1.1. The evidence for formation of a KP-HSA complex and determination of the binding sites in the high affinity binding site (HAS)

The interaction of KP with HSA was investigated by monitoring tryptophanyl (Trp 214) residue fluorescence quenched by KP. In order to analyze the interaction of KP with HSA in the HAS of protein fluorescence spectra of $8 \times 10^{-6} \mathrm{~mol} / \mathrm{L} \mathrm{HSA}$ in the presence of increasing amounts of KP (concentration of KP $5 \times 10^{-6} \div 4 \times$ $10^{-4} \mathrm{~mol} / \mathrm{L}$ ) at $\lambda_{\text {ex }}=295 \mathrm{~nm}$ were recorded (the inset in Fig. 1).

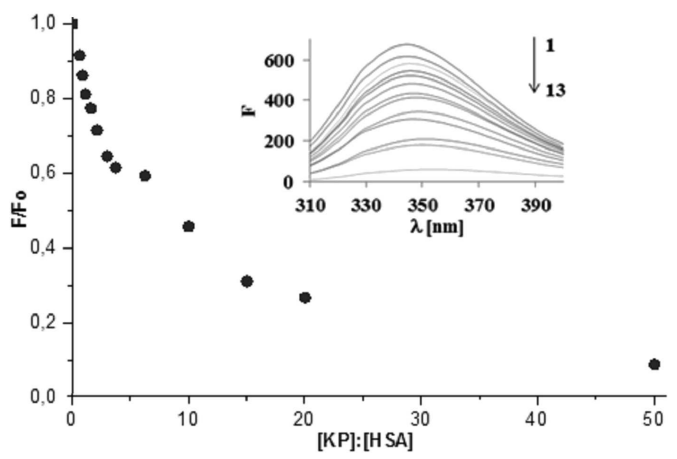

Fig. 1. Quenching curve of HSA $\left(8 \times 10^{-6} \mathrm{~mol} / \mathrm{L}\right)$ in the presence of $\mathrm{KP}\left(5 \times 10^{-6} \div 4 \times 10^{-4} \mathrm{~mol} / \mathrm{L}\right)$, $[\mathrm{KP}]:[\mathrm{HSA}] \quad 0.625: 1 \div 50: 1$ (in the inset fluorescence emission spectra of $\mathrm{HSA}$ in the presence of KP), $\lambda_{\mathrm{ex}}=$ $295 \mathrm{~nm}$.

A binding of small molecules to HSA induces changes in protein conformation and consequently its fluorescence. With the increase of KP concentration the decrease in albumin's fluorescence has been observed. The quenching of protein fluorescence by the KP may indicate drug-protein interaction. A variety of molecular interactions can result in quenching, including excitedstate reactions, molecular rearrangements, ground-state complex formation and collisional quenching [22]. According to Förster's nonradiative energy transfer theory, the energy transfer can occur only when the fluorescence emission spectra of the donor and the absorption spectra of the acceptor have enough overlap and the distance between donor and acceptor should not exceed $10 \mathrm{~nm}$ $[10,23]$.

In order to determine the location of KP binding site(s) in HSA $295 \mathrm{~nm}$ wavelength was used. Excitation of serum albumin at $295 \mathrm{~nm}$ produces fluorescence of the tryptophan residue. HSA has one tryptophanyl residue Trp 214 in Sudlow's binding site I (subdomain IIA). Figure 1 presents quenching curves of HSA $\left(8 \times 10^{-6} \mathrm{~mol} / \mathrm{L}\right)$ in the presence of KP at increasing concentration. Analysis of the quenching curves of HSA fluorescence shows the decrease fluorescence of albumin excited at $295 \mathrm{~nm}$. The maximum of quenching at saturation reaches $90 \%$. Quenching of HSA fluorescence by KP excited at $\lambda_{\mathrm{ex}}=$ $295 \mathrm{~nm}$ (Fig. 1) indicate that KP interacts with Trp 214 residue and accepts its energy. In order to specify the KP binding in hydrophobic regions of HSA competitive studies with ANS and DNSA have been conducted. ANS and DNSA are fluorescent probes with the highest affinity towards subdomain IIIA [24] and IIA, respectively. ANS also binds in subdomain IIA, however its predominant affinity to subdomain IIIA allows to use it at low concentration as a selective probe for Sudlow's site II [25]. The ability of the ligand displacement by the fluorescent probes from characteristic of these probes sites (IIIA, IIA) is determined by the strength of binding and specific binding sites for these ligands [9]. It means that if specific ligand binding sites will be common for ligand and fluorescent probes the ligand is able to release him from this place.

Specificity and relative strength of drug binding to the binding site can be determined by the percentage displacement of drug [9]:

$$
\frac{F_{1}-F_{n}}{F_{1}} \times 100 \%
$$

where $F_{1}$ is the fluorescence of probe and HSA in the absence of the quencher (ligand); $F_{n}$ is the fluorescence of probe and HSA in the presence of increasing concentration of the quencher (ligand).

ANS and DNSA excited at $\lambda_{\text {ex }}=295$ and $360 \mathrm{~nm}$ and DNSA excited at $350 \mathrm{~nm}$ respectively, show fluorescent abilities when they are bound to protein. Displacement of ANS and DNSA by KP is seen as the decrease of the probe fluorescence. A plot of percentage displacement vs. KP is shown in Fig. 2.

Slight changes in ANS $\left(\lambda_{\mathrm{ex}}=360 \mathrm{~nm}\right)$ and the significant changes in ANS $\left(\lambda_{\mathrm{ex}}=295 \mathrm{~nm}\right)$ fluorescence in the presence of ketoprofen at increasing concentration (Fig. 2) suggest that KP binds in subdomain IIIA. KP is used to photolabel the binding region of site II [26]. ANS is displaced by KP in almost $90 \%$. Studies on KP binding to HSA showed that the aryl propionic acid NSAIDs containing both anionic and hydrophobic groups bind strongly to the subdomain IIIA (diazepam site) [6]. A slight change in DNSA fluorescence in the presence of $\mathrm{KP}$ at increasing concentration and lower percentage displacement of KP (20\%) suggest that KP does not bind in subdomain IIA. Hydrophobic subdomain IIIA of HSA is the primary binding site of KP. 


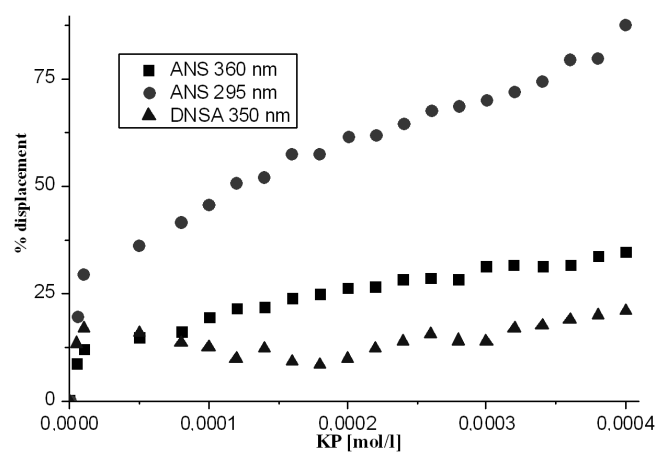

Fig. 2. The percentage displacement of ANS $(8 \times$ $\left.10^{-6} \mathrm{~mol} / \mathrm{L}\right)$ and DNSA $\left(4 \times 10^{-5} \mathrm{~mol} / \mathrm{L}\right)$, by $\mathrm{KP}$ $\left(5 \times 10^{-6}\right.$ to $\left.4 \times 10^{-4} \mathrm{~mol} / \mathrm{L}\right)$.

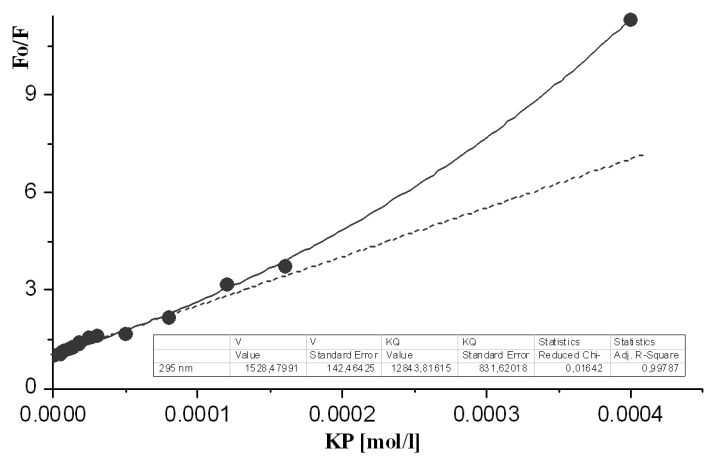

Fig. 3. The Stern-Volmer plots of $F_{o} / F$ vs. [KP] concentration for KP-HSA complex, $\lambda_{\mathrm{ex}}=295 \mathrm{~nm}$.
Fluorescence intensity data was analyzed according to the Stern-Volmer equation (2.1) [15] by plotting $\frac{F_{0}}{F}$ vs. [KP] (Fig. 3).

The Stern-Volmer plot is characteristic for the behavior of KP molecules on interacting with HSA fluorescence (Fig. 3). Two ranges of KP concentration have been observed. KP molecules receive the energy from excited HSA fluorophores, the drug molecule moves dislodged and makes space for molecules of another ligand. A similar effect was observed for phenylbutazone-serum albumin and aspirin-serum albumin complexes [27]. The Stern-Volmer plot showed a linear relationship between fluorescence intensity and quencher concentration (KP) below $8 \times 10^{-5} \mathrm{~mol} / \mathrm{L}$ (Fig. 3 ). It points to the dynamic mechanism of quenching. At high concentration of KP, above $8 \times 10^{-5} \mathrm{~mol} / \mathrm{L}$, positive deviation from linearity suggests a more complex quenching process, probably an additional presence of a static component in the quenching mechanism [16]. This confirms the Eftink and Ghiron conclusions who said that in the case of upward curving plot, the static and collisional quenching modes can be dissected [28]. The static quenching is often observed if the fluorophore is adjacent to ketoprofen at the moment of excitation [29].

On the basis of modified form of the Stern-Volmer Eq. (2.2) dynamic $\left(K_{\mathrm{Q}}\left[\mathrm{M}^{-1}\right]\right)$ and static $\left(V\left[\mathrm{M}^{-1}\right]\right)$ constants can be obtained [16]. Quenching constants have been calculated on the basis of non-linear regression using the computer program OriginPro 8.5 and collected in Table Ia.

TABLE I

Values of the binding parameters obtained on the basis of Eqs. (2.2)-(2.6) in KP-HSA complex.

\begin{tabular}{lc|c|c|c|c|c|c}
\hline \hline & $\begin{array}{c}\text { Binding parameters } \\
\text { obtained by the } \\
\text { following methods: }\end{array}$ & $\begin{array}{c}K_{\mathrm{Q}} \times 10^{-4} \\
{\left[\mathrm{M}^{-1}\right]}\end{array}$ & $\begin{array}{c}V \times 10^{-4} \\
{\left[\mathrm{M}^{-1}\right]}\end{array}$ & $\begin{array}{c}K_{\mathrm{a}} \times 10^{-4} \\
{\left[\mathrm{M}^{-1}\right]}\end{array}$ & $f_{\mathrm{a}}$ & $N$ & $n_{\mathrm{H}}$ \\
\hline a) & $\begin{array}{c}\text { Stern-Volmer (Eq. (2.2)) } \\
\lambda_{\text {ex }}=295 \mathrm{~nm}\end{array}$ & 1.28 & 0.15 & - & - & - & - \\
\hline b) & $\begin{array}{c}\text { modified Stern-Volmer (Eq. (2.3)) } \\
\lambda_{\text {ex }}=295 \mathrm{~nm}\end{array}$ & 1.85 & - & - & 1.09 & - & - \\
\hline c) & $\begin{array}{c}\text { Scatchard (Eq. (2.4)) } \\
\lambda_{\text {ex }}=295 \mathrm{~nm}\end{array}$ & - & - & 2.69 & - & - & - \\
\hline $\mathrm{d})$ & $\begin{array}{c}\text { Klotz (Eq. (2.5)) } \\
\lambda_{\mathrm{ex}}=295 \mathrm{~nm}\end{array}$ & - & - & 2.15 & - & 0.95 & - \\
\hline e) & $\begin{array}{c}\text { Hill (Eq. (2.6)) } \\
\lambda_{\mathrm{ex}}=295 \mathrm{~nm}\end{array}$ & - & - & 1.59 & - & - & 0.84 \\
\hline
\end{tabular}

When tryptophanyl residue was excited ketoprofen quenches HSA fluorescence with the following dynamic $\left(K_{\mathrm{Q}}\right)$ and static $(V)$ quenching constants (Eq. (2.2)) $K_{\mathrm{Q}}=1.28 \times 10^{4} \mathrm{M}^{-1}$ and $V=0.15 \times 10^{4} \mathrm{M}^{-1}$, respectively. On the basis of Eq. (2.3) [17] the modified Stern-Volmer plots have been drawn (Fig. 4).
The values of fractional accessibility of the fluorophore $f_{\mathrm{a}}$ and quenching constant $K_{\mathrm{Q}}\left[\mathrm{M}^{-1}\right]$ were obtained from values of intercept and slope, respectively (Table $\mathrm{Ib}$ ). The value of $K_{\mathrm{Q}}$ obtained for KP-HSA excited at $\lambda_{\mathrm{ex}}=$ $295 \mathrm{~nm}$ is $1.85 \times 10^{4} \mathrm{M}^{-1}$ (Fig. 4). $f_{\mathrm{a}}$ determined for KP-HSA complex is 1.09 . The fractional accessibility is 
equal to or higher than 1 . This means that accessibility of the fluorophore to the quencher is total. When the distance between excited fluorophore and ligand was smaller, the growth of accessibility of fluorophore was observed.

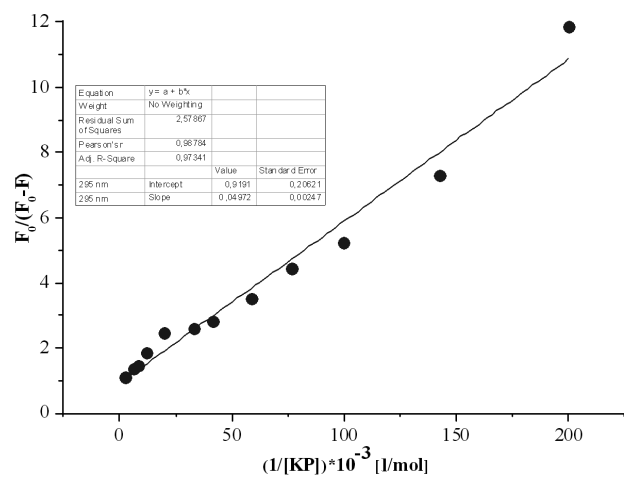

Fig. 4. Modified Stern-Volmer plots in KP-HSA complex, $\lambda_{\text {ex }}=295 \mathrm{~nm}$.

\subsubsection{Binding parameters}

The binding of KP to several classes of sites on protein molecule has been represented by the Scatchard (Eq. (2.4), [18]), Klotz (Eq. (2.5), [19]) and Hill (Eq. (2.6), [20]) methods by plotting $\frac{r}{\left[\mathrm{~L}_{\mathrm{f}}\right]}$ vs. $r, \frac{1}{r}$ vs. $\frac{1}{\left[\mathrm{~L}_{\mathrm{f}}\right]}$ and $\lg \left(\frac{Q}{1-Q}\right)$ vs. $\lg \left(L_{\mathrm{f}}\right)$, respectively. On the basis of Eqs. (2.4)-(2.6) Figs. 5-7 have been drawn.

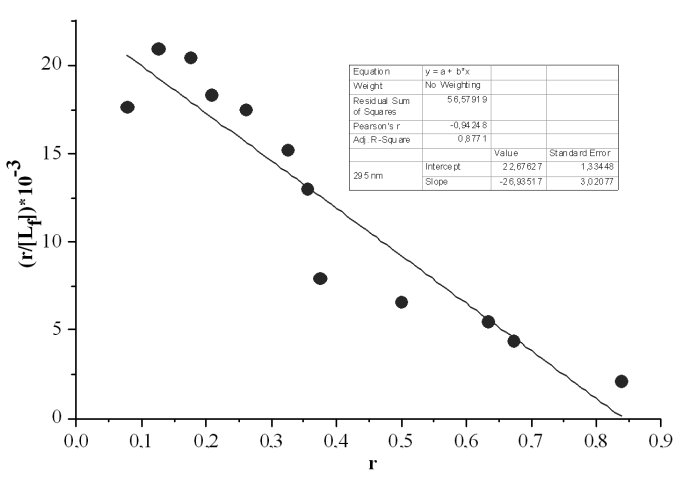

Fig. 5. Scatchard plots of $[r] /\left[\mathrm{L}_{\mathrm{f}}\right]$ vs. $r$ for KP-HSA complex, $\lambda_{\mathrm{ex}}=295 \mathrm{~nm}$.

Binding parameters obtained from slope and intercept of equation have been collected in Table Ic-e.

The plots obtained on the basis of Eqs. (2.4)-(2.6) show linear dependence for KP-HSA complex. The run of these plots is specific for one-site model and indicates the existence of one independent class of KP binding site in this subdomain.

On the basis of modified by Hiratsuka Scatchard Eq. (2.4) [18] (Fig. 5) the following association constant has been calculated: $K_{\mathrm{a}}=2.69 \times 10^{4} \mathrm{M}^{-1}$ (Table Ic). Similarly, binding parameters have been calculated using the Klotz (Eq. (2.5), Fig. 6) and Hill (Eq. (2.6),

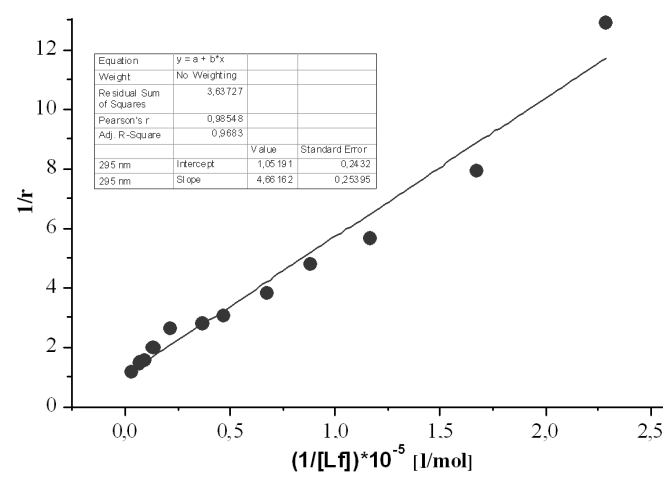

Fig. 6. Klotz plots of $1 / r$ vs. $1 /\left[\mathrm{L}_{\mathrm{f}}\right]$ for KP-HSA complex, $\lambda_{\mathrm{ex}}=295 \mathrm{~nm}$.

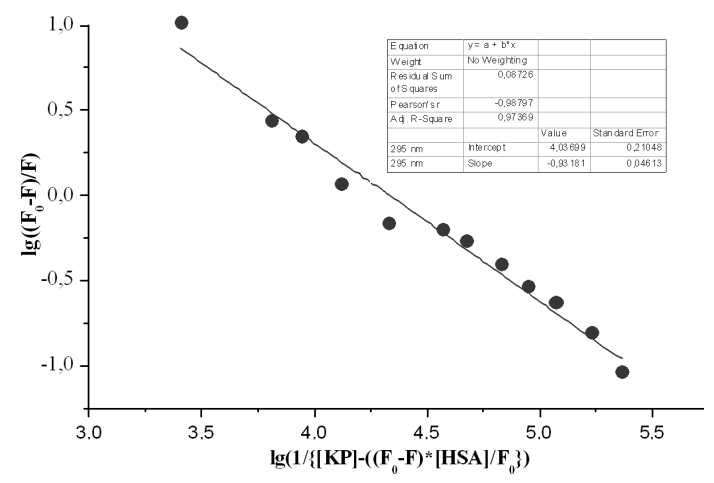

Fig. 7. Hill's plots of $\lg (Q /(1-Q))$ vs. $\lg \left(\mathrm{L}_{\mathrm{f}}\right)$ for KP-HSA complex, $\lambda_{\mathrm{ex}}=295 \mathrm{~nm}$.

Fig. 7) equations. For Eq. (2.5) $K_{\mathrm{a}}=2.15 \times 10^{4} \mathrm{M}^{-1}$ and $n=0.95$ values were collected in Table Id. On the basis of Hill method (Eq. (2.6)) $K_{\mathrm{a}} \approx 1.6 \times 10^{4} \mathrm{M}^{-1}$ and $n_{\mathrm{H}} \approx 0.8$ values were collected in Table Ie.

The analysis of binding parameters shows deviations in the values obtained from the literature and calculated using different methods. This fact may be explained by Bhattacharya work in which the author described the influence of albumin's origin and the techniques used achieved the value of binding constants [30]. Scatchard's formula shows the relationship between $\frac{r}{\left[\mathrm{~L}_{\mathrm{f}}\right]}$ and the bound fraction of ligands while Klotz's and Hill's equations represent the dependence between $\frac{1}{r}$ and $\lg \left(\frac{Q}{1-Q}\right)$, respectively, and a free fraction of ligand. If a greater part of ligand molecules is bound, the sum of analytical errors committed during the experiment strongly affects data connected with a free fraction of ligand. This probably contributes to the occurrence of differences between the values of binding parameters obtained using these three methods. The values of association constant obtained by using three methods of processing the experimental data attest a strong interaction between KP and HSA. The order of $K_{\mathrm{a}}$ values $\left(10^{4}\right)$ is consistent with non-covalent interactions [31].

Binding of KP to HSA does not show cooperativeness in ligand binding. Hill coefficient $n_{\mathrm{H}}$ us lower than 
1 (Table Ie), which suggests that the binding of one molecule of KP to serum albumin decreases affinity of $\mathrm{KP}$ for another molecule.

\section{2. ${ }^{1} H N M R$ studies}

3.2.1. The evidence of formation of a KP-HSA complex in the low affinity binding site (LAS). Analysis of the chemical shifts

Figure 8 shows proton magnetic resonance spectrum and ${ }^{1}$ HNMR chemical structure of ketoprofen.

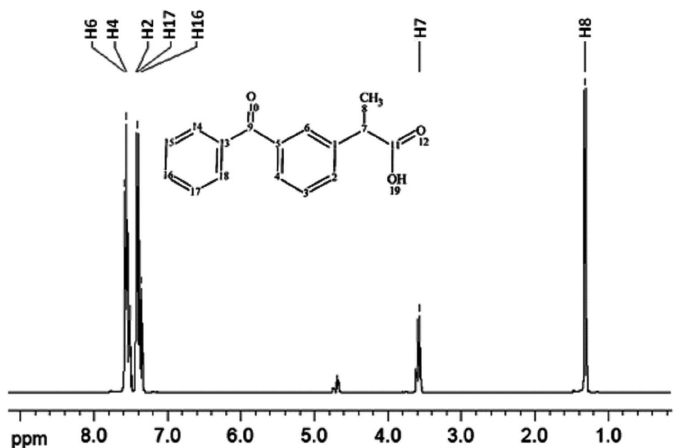

Fig. 8. ${ }^{1} \mathrm{HNMR}$ spectrum (400 $\left.\mathrm{MHz}\right)$ of ketoprofen and chemical structure of drug.

On the basis of the chemical shift $(\sigma)$ of H16 and H8 KP proton resonance signals the dependence of $\sigma$ vs. [KP]:[HSA] was plotted (Fig. 9a,b, respectively).

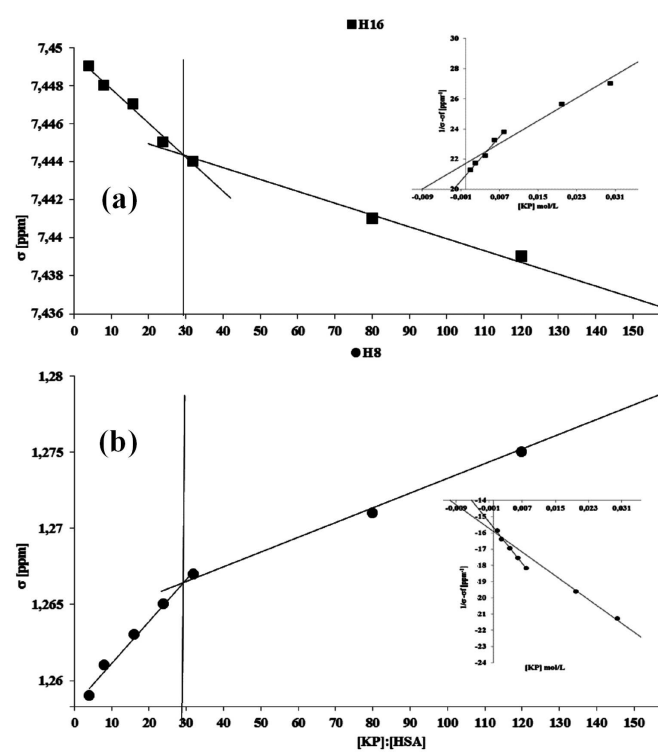

Fig. 9. Chemical shifts of proton (a) H16, (b) H8 resonance of $\mathrm{KP}$ at increasing [KP]:[HSA] molar ratio. In the inset $1 /\left(\sigma-\sigma_{\mathrm{f}}\right)$ vs. [KP] dependence for (a) H16, (b) H8. HSA $2.5 \times 10^{-4} \mathrm{~mol} / \mathrm{L}, \mathrm{KP} 2 \times 10^{-3} \div$ $4 \times 10^{-2} \mathrm{~mol} / \mathrm{L}$

With the increase of $[\mathrm{KP}]:[\mathrm{HSA}]$ molar ratio the slope of the plot $\sigma$ vs. [KP]:[HSA] changes (Fig. 9). Changes in the chemical shift of the bound and unbound KP indicate structural alterations in its environment and mean that KP interacts with HSA and locates in its low affinity binding site. Below [KP]:[HSA] molar ratio 29:1 significant changes of the chemical shifts were observed for both aromatic and aliphatic protons in the presence of HSA. Above the molar ratio [KP]:[HSA] 29:1 only a small change of ${ }^{1} \mathrm{HNMR}$ chemical shifts of $\mathrm{H} 16$ and $\mathrm{H} 8$ of $\mathrm{KP}$ protons was observed. The position of aromatic H16 (Fig. 9a) and methyl H8 (Fig. 9b) resonance signals of $\mathrm{KP}$ shifts with the increase of the molar ratio $[\mathrm{KP}]:[\mathrm{HSA}]$ in the presence of HSA towards a higher (Fig. 9a) and lower (Fig. 9b) fields, respectively. The upfield shift of aromatic $\mathrm{H} 16$ proton resonances of KP can be explained by the magnetic shielding effect in the KP-HSA complex formed with serum albumin and points to the increase in electron density in the surroundings of H16 proton resonance signal of KP. This observation is similar to the results obtained for Phe-ASA-HSA ternary complex [27].

The reverse phenomenon around the H8 KP protons can be explained by the magnetic deshielding effect in the complex formed with HSA and points to a decrease in electron density in the surroundings of KP aromatic proton (H8). Moreover, the deshielding effect of the aromatic protons of KP may be caused by the hydrogen bonds formed by $\mathrm{NH}_{2}, \mathrm{OH}$ and $\mathrm{SH}$ groups in the structure of the albumin. This is in agreement with the studies described in previous study $[11,32]$. Furthermore, above the molar ratio KP:HSA 29:1 the position of $\mathrm{H} 16$ and $\mathrm{H} 8$ resonance signals still shifted towards a higher and lower field, respectively.

Upfield shift of aromatic proton $\mathrm{H} 16$ is a result of the $\pi-\pi$ interactions between the aromatic ring of $\mathrm{KP}$ and the aromatic amino acid of the HSA polypeptide chain (Fig. 9a). Because the plot $\sigma$ vs. [KP]:[HSA] above the molar ratio 29:1 does not reach a plateau it is noteworthy that KP-HSA complex is not saturated and HSA is able to bind new molecules of KP. This phenomenon can arise from the fact that KP molecules locate in two classes of the LAS. The chemical shifts $\sigma$ [ppm] of aromatic H16 and methyl $\mathrm{H} 8$ proton resonances of KP in the KP-HSA system were compared with those of KP in the buffer and $\Delta \sigma[\mathrm{ppm}]$ values were calculated. The analysis of $\Delta \sigma$ for both aromatic proton $\mathrm{H} 16\left(\Delta \sigma_{\mathrm{H} 16}=0.029 \mathrm{ppm}\right)$ and the methyl $\mathrm{H} 8\left(\Delta \sigma_{\mathrm{H} 8}=0.041 \mathrm{ppm}\right.$, Fig. 9b) group of ketoprofen in the complex with HSA suggests a greater participation of the methyl group in the complex with protein. The aliphatic chain of KP plays an important role in the formation of a KP-HSA complex in the secondary low affinity binding site. The selective changes in the chemical shifts can suggest an unequal participation of particular elements of KP molecule in the formation of the KP-HSA complex.

\subsubsection{Analysis of association constants}

According to the method described in previous paper [33] and on the basis of Eq. (2.7) dissociation constant $K_{\mathrm{d}}\left[\mathrm{M}^{-1}\right]$ which finally was used to determine the association constant $K_{\mathrm{a}}\left[\mathrm{M}^{-1}\right]$ was calculated. As seen in 
Fig. 9 (the insets) the slope of the plot $1 /\left(\sigma-\sigma_{\mathrm{f}}\right)$ vs. [KP] changes. It means that KP molecules locate in two classes of the LAS. For each classes association constants $K_{\mathrm{a}}\left[\mathrm{M}^{-1}\right]$ were determined. The obtained parameters were collected in Table II.

\section{TABLE II}

Association constant $K_{\mathrm{a}}$ for the independent classes of low affinity binding sites of KP in HSA macromolecule.

\begin{tabular}{c|c|c|c}
\hline \hline \multicolumn{2}{c|}{$K_{\mathrm{aI}} \times 10^{-2}\left[\mathrm{M}^{-1}\right]$} & \multicolumn{2}{c}{$K_{\mathrm{aII}} \times 10^{-2}\left[\mathrm{M}^{-1}\right]$} \\
\hline $\mathrm{H} 16$ & $\mathrm{H} 8$ & $\mathrm{H} 16$ & $\mathrm{H} 8$ \\
\hline 0.17 & 0.21 & 0.09 & 0.11
\end{tabular}

Association constants for KP-HSA complex in the primary binding site are $0.17 \times 10^{2} \mathrm{M}^{-1}$ and $0.21 \times 10^{2} \mathrm{M}^{-1}$, for aromatic proton $\mathrm{H} 16$ and methyl $\mathrm{H} 8$ group of KP, respectively. In the secondary binding sites $K_{\mathrm{a}}$ are equal to $0.09 \times 10^{2} \mathrm{M}^{-1}(\mathrm{H} 16)$ and $0.11 \times 10^{2} \mathrm{M}^{-1}$ (H8). These constants are three orders of magnitude lower than those estimated on the basis of the fluorescence measurements (Table I). ${ }^{1} \mathrm{HNMR}$ technique is characterized by fast exchange between the free and bound drug molecules. Binding sites of ketoprofen observed with the use of ${ }^{1} \mathrm{HNMR}$ technique are impossible to be observed by spectrofluorimetry because they are probably located on the surface of the albumin. Therefore constants determined by using ${ }^{1} \mathrm{HNMR}$ are lower than that determined from fluorescence study which allows to characterize the high affinity inner binding sites in the hydrophobic pockets containing Trp 214 and Tyr 401/411. The association constants calculated for a particular class of binding sites on the basis of chemical shifts of observed H16 and H8 proton resonances of KP have similar values (Table II). This phenomenon probably indicates an equal participation of both rings of KP engaged in the interaction with albumin. The value of the association constants estimated for both classes of binding sites indicates weak binding KP-HSA complex reflecting a very fast exchange between free and bound drug molecule.

\section{Conclusions}

Determination of albumin binding parameters could be of pharmacological relevance in order to help in evaluating the proper therapeutic dose of the specific treatment. This work is an example of study wherein the interaction of HSA with a nonsteroidal anti-inflammatory drug (KP) has been investigated by fluorescence and ${ }^{1} \mathrm{HNMR}$ spectroscopy. Spectrofluorescence and proton nuclear magnetic resonance spectroscopy have been used to characterize the HAS and LAS of HSA. The fluorimetric results revealed that the hydrophobic interaction was a predominant intermolecular force for stabilizing the complex. The authors indicated that KP binds to subdomain IIIA of HSA with a high affinity to tryptophan residue by hydrophobic interaction. To obtain association constants in the high affinity binding sites Scatchard, Klotz and Hill methods were used. The use of ${ }^{1} \mathrm{HNMR}$ spectroscopy allowed to conclude that ketoprofen has a relatively low affinity to albumin. The analysis of both chemical shift and association constants showed that the molecules of ketoprofen may approach the surface of the protein and form weak interactions in the low affinity binding sites.

Simultaneous use of both fluorescence and ${ }^{1} \mathrm{HNMR}$ methods seems to be necessary. It allows for a thorough analysis of strong and weak interactions that occur during formation of a KP-HSA complex.

\section{Acknowledgments}

The research was financially supported by the grants KNW-1-016/N/2/0 and KNW-1-077/P/2/0 from Medical University of Silesia, Poland.

\section{References}

[1] A. Gibofsky, HSS J. 2, 30 (2006).

[2] D. Aletaha, T. Neogi, A.J. Silman, J. Funovits, D.T. Felson, C.O. Bingham, N.S. Birnbaum, G.R. Burmester, V.P. Bykerk, M.D. Cohen, B. Combe, K.H. Costenbader, M. Dougados, P. Emery, G. Ferraccioli, J.M.W. Hazes, K. Hobbs, T.W.J. Huizinga, A. Kavanaugh, J. Kay, T.K. Kvien, T. Laing, P. Mease, H.A. Ménard, L.W. Moreland, R.L. Naden, T. Pincus, J.S. Smolen, E. StanislawskaBiernat, D. Symmons, P.P. Tak, K.S. Upchurch, J. Vencovský, F. Wolfe, G. Hawker, Arthritis Rheum. 62, 2569 (2010).

[3] A. Diaz-Borjón, Drugs Aging 26, 273 (2009).

[4] B. Maziěres, Drugs RD 6, 337 (2005).

[5] M. Otagiri, Drug Metab. Pharmacokinet. 20, 309 (2005).

[6] U. Kragh-Hansen, V.T.G. Chuang, M. Otagiri, Biol. Pharm. Bull. 25, 695 (2002).

[7] S. Sugio, A. Kashima, S. Mochizuki, M. Noda, K. Kobayashi, Protein Eng. 12, 439 (1999).

[8] A. Dugaiczyk, S.W. Law, O.E. Dennison, Proc. Natl. Acad. Sci. USA 79, 71 (1982).

[9] G. Sudlow, D.J. Birkett, D.N. Wade, Mol. Pharmacol. 11, 824 (1975).

[10] A. Sułkowska, M. Maciążek-Jurczyk, B. Bojko, J. Równicka-Zubik, I. Zubik-Skupień, E. Temba, D. Pentak, W.W. Sułkowski, J. Mol. Struct. 881 , 97 (2008).

[11] M. Maciążek-Jurczyk, A. Sułkowska, J. Równicka-Zubik, B. Bojko, A. Szkudlarek-Haśnik, M. Knopik, W.W. Sułkowski, J. Mol. Struct. 993, 302 (2011).

[12] V.T.G. Chuang, A. Kuniyasu, H. Nakayama, Y. Matsushita, S. Hirono, M. Otagiri, Biochem. Biophys. Acta 1434, 18 (1999).

[13] H. Watanabe, S. Tanase, K. Nakajou, T. Maruyama, U. Kragh-Hansen, M. Otagiri, Biochem. J. 349, 813 (2000).

[14] E.P. Kirby, in: Fluorescence Instrumentation and Methodology, Eds. R.F. Steinem, I. Weinryb, Plenum Press, New York 1971, p. 31.

[15] O. Stern, M. Volmer, Phys. Z. 20, 183 (1919). 
[16] A. Varlan, M. Hillebrand, Molecules 15, 3905 (2010).

[17] S.S. Lehrer, Biochemistry 10, 3254 (1971).

[18] G. Scatchard, Ann. N.Y. Acad. Sci. 51, 660 (1949).

[19] I.M. Klotz, D.L. Hunston, Biochemistry 10, 3065 (1971).

[20] A.V. Hill, J. Physiol. 40, iv (1910).

[21] Y. Xu, T. Seto, P. Tang, L. Firestone, Biophys. J. 78,746 (2000).

[22] J.R. Lakowicz, Principles of Fluorescence Spectroscopy, Plenum Press, New York, 1983.

[23] L. Stryer, Science 162, 526 (1968).

[24] L.A. Bagatolli, S.C. Kivatinitz, G.D. Fidelio, J. Pharm. Sci. 85, 1131 (1996).

[25] B. Bojko, A. Sułkowska, M. Maciążek-Jurczyk, J. Równicka, W.W. Sułkowski, J. Mol. Struct. 924-926, 332 (2009).

[26] K. Kaneko, V.T.G. Chuang, T. Ito, A. Suenaga, H. Watanabe, T. Maruyama, M. Otagiri, Die Pharmazie 67, 414 (2012).
[27] M. Maciążek-Jurczyk, A. Sułkowska, B. Bojko, J. Równicka, W.W. Sułkowski, Spectrochim. Acta Mol. Biomol. Spectrosc. 82, 181 (2011).

[28] M.R. Eftink, C.A. Ghiron, J. Phys. Chem. 80, 486 (1976).

[29] J. Seetharamappa, B.P. Kamat, Chem. Pharm. Bull. 52, 1053 (2004).

[30] A.A. Bhattacharya, S. Curry, N.P. Franks, J. Biol. Chem. 275, 38731 (2000).

[31] H. Aki, M. Yamamoto, J. Pharm. Pharmacol. 41, 674 (1989).

[32] A. Sułkowska, M. Maciążek, J. Równicka, B. Bojko, D. Pentak, W.W. Sułkowski, J. Mol. Struct. 834-836, 162 (2007).

[33] M. Maciążek-Jurczyk, A. Sułkowska, J. Równicka-Zubik, A. Szkudlarek-Haśnik, W.W. Sułkowski, Spectrosc. Lett. 45, 365 (2012). 